\title{
The comprehensive impact on human body induced by resolution of growth hormone excess
}

\author{
Zhaoyun Zhang 1,2,*, Qin Li, 2,*, Wenqiang He 2,3, *, Huijia Qiü,3,*, Hongying Ye 1,2,*, Yongfei Wang 2,3,*, \\ Ming Shen ${ }^{2,3}$, Min He ${ }^{1,2}$, Yifei Yu1,2, Xuefei Shou ${ }^{2,3}$, Chuanxin Huang ${ }^{4}$, Huan Yu5, Guoqian Huang ${ }^{6}$, Weijun Tang ${ }^{7}$, \\ Daoying Geng ${ }^{7}$, Chaowei Fu' ${ }^{8}$, Congjin Liu' ${ }^{9}$, Zengyi Ma², ${ }^{2,}$ Zhao Ye ${ }^{2,3}$, Qilin Zhang ${ }^{2,3}$, Yichao Zhang ${ }^{2,3}$, \\ Yue Shen ${ }^{2,3}$, Yeping Yang ${ }^{1,2}$, Meng Wang ${ }^{1,2}$, Xingdang Liu' ${ }^{9}$, Yun Lu', Renming Hư ${ }^{1,2}$, Ying Mao ${ }^{2,3}$, \\ Liangfu Zhou ${ }^{2,3}$, Yiming Li ${ }^{1,2}$, Shiqi Li' ${ }^{2,3}$, Nicholas A Tritos ${ }^{10}$ and Yao Zhao ${ }^{2,3}$ \\ 'Department of Endocrinology and Metabolism, Huashan Hospital, Shanghai Medical College, Fudan University, Shanghai, China, \\ ${ }^{2}$ Shanghai Pituitary Tumor Center, Shanghai, China, ${ }^{3}$ Department of Neurosurgery, Huashan Hospital, Shanghai Medical College, \\ Fudan University, Shanghai, China, ${ }^{4}$ Shanghai Institute of Immunology, Shanghai Jiao Tong University School of Medicine, Shanghai, \\ China, ${ }^{5}$ Department of Neurology, ${ }^{6}$ Department of Echocardiography, ${ }^{7}$ Department of Radiology, Huashan Hospital, Shanghai Medical \\ College, Fudan University, Shanghai, China, ${ }^{8}$ Department of Epidemiology, School of Public Health, Key Laboratory \\ of Public Health Safety of Ministry of Education, Fudan University, Shanghai, China, ${ }^{9}$ Department of Nuclear \\ Medicine, Huashan Hospital, Shanghai Medical College, Fudan University, Shanghai, China, and ${ }^{10}$ Neuroendocrine \\ Unit, Massachusetts General Hospital and Harvard Medical School, Boston, Massachusetts, USA \\ Correspondence \\ should be addressed \\ to Z Zhang or Y Zhao \\ Email

\begin{abstract}
Context: Chronic excess of growth hormone $(\mathrm{GH})$ often leads to systemic complications. The reversibility of these complications after $\mathrm{GH}$ resolution is not fully understood.

Objective: To investigate when and to what extent will the comorbidities be ameliorated.

Design: We conducted a prospective study comprising 24 patients with acromegaly, who achieved remission after transsphenoidal surgery. The dynamic changes of endocrine, cardiovascular, respiratory, sleep, bone and morphology parameters were evaluated at enrollment and 1 week, 1 month, 3 months, 6 months and 12 months after surgery. Results: Random GH dropped by $98.4 \%$ at the first day postoperatively. IGF-I index dropped by $50 \%$ and $64 \%$ at 1 week and 1 month respectively and remained unchanged onwards. Glucose metabolism improved significantly at 1 week and stabilized at 1 month. Testosterone in male patients recovered to normal range since 1 month. Systolic blood pressures dropped markedly at 3 months while diastolic blood pressures fell mildly at later visits. Abnormal lung function showed no improvement. The decrease of bone formation and resorption markers occurred at 1 week and 3 months, respectively. At 1 month, the tongue area declined while the airway volume increased significantly, accompanied with improved obstructive sleep apnea syndrome. Extremities, lips and nasal ala became smaller since 1 week. Liver, kidney and spleen volumes declined by $6.4,15.9,9.2 \%$, respectively at 1 month. The volumes of pancreas and adrenal showed no change.

Conclusions: The rapid resolution of excessive GH led to the reversible changes of systemic comorbidities in a timedependent and organ-specific manner.

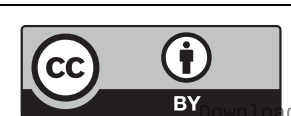
This work is licensed under a Creative Commons Attribution 4.0 International License. 


\section{Introduction}

Growth hormone (GH) plays critical roles in human physiology. However, chronic exposure to endogenous excessive GH leads to extensive effects on body organs and results in systemic complications, including endocrine, cardiovascular, respiratory and neoplastic sequelae, which are mostly seen in acromegaly $(1,2,3,4,5)$. It has long been recognized that chronic GH excess induces systemic complications. However, after the resolution of excessive GH (surgical remission or medical control of GH-secreting adenomas), it is not fully understood whether the impairments in anatomy and function of major organs are reversible. As this relates to the clinical management, it is important to elucidate the time course of anatomic and physiologic changes occurring after biochemical remission. However, it is difficult to investigate it, due to the involvement of multiple organs and lacking of animal models for experiments. Ingeniously, subjects with acromegaly cured by surgery are an extraordinary model to investigate the pathophysiology of GH and insulinlike growth factor 1 (IGF-I) actions on human organs and systems. Clinical manifestations of acromegaly develop insidiously over time. Even newly diagnosed patients with acromegaly usually present with multiple comorbidities (1). Thorough investigation of patients with acromegaly experiencing remission from surgery helps us better understand to what extent these comorbidities will be ameliorated and how fast these changes will occur.

To answer these questions, there are many retrospective studies, which mostly examined a limited number of cardiovascular, respiratory or other systems over irregular follow-up periods. Few studies prospectively examined the dynamic changes of systemic comorbidities after surgery $(6,7)$. Here, we present our prospective study in which endocrine, cardiovascular, respiratory, sleep, bone and morphological parameters have been examined in patients with newly diagnosed acromegaly, both before the surgery and over a one-year period after surgical remission. We report that rapid remission of excessive GH-induced ameliorations in an organ- and time-dependent manner, which not only answered the above clinical questions, but also may inspire deeper understanding about the complex physiological and pathophysiological effects of GH.

\section{Subjects and methods}

\section{Patients and study design}

We prospectively recruited 48 newly diagnosed patients with acromegaly, who underwent transsphenoidal microscopic adenomectomy and were regularly followed from June 2010 to June 2012 in Huashan Hospital, Fudan University. Acromegaly was diagnosed based on clinical findings, IGF-I level above normal range, failure of suppression of $\mathrm{GH}<1.0 \mu \mathrm{g} / \mathrm{L}$ during oral glucose tolerance test (OGTT), radiological and pathological confirmation (8). Patients were considered cured when either a GH nadir $\left(\mathrm{GH}_{\mathrm{n}}\right)<0.4 \mu \mathrm{g} / \mathrm{L}$ or random $\mathrm{GH}<1.0 \mu \mathrm{g} / \mathrm{L}$ in addition to normalized IGF-I on the last follow-up (8). The study protocol (www.ClinicalTrials. gov Identifier: NCT01368133) was approved by the ethical committees of Huashan Hospital. All patients gave written informed consent. Evaluations prior to and one week, one month, three months, six months and one year after surgery were performed. At one-year visit, 24 patients were considered to be cured and were included in the final analysis.

\section{Systemic examinations}

At each visit, physical examinations were performed and a detailed history was obtained. Trained staff who were blinded to the study helped to take the measurements. Hand volumes, foot volumes, lip thickness, nasal ala width and joint perimeters were measured. After overnight fasting, subjects underwent morning blood sampling for hormone workup (IGF-I, $\mathrm{FT}_{3}, \mathrm{FT}_{4}$, TSH, $\mathrm{LH}, \mathrm{FSH}, \mathrm{ACTH}$, progesterone, testosterone, estrogen, prolactin, cortisol), glycated hemoglobin $\left(\mathrm{HbA}_{1 \mathrm{C}}\right)$, lipid profiles, bone turnover markers, hepatic and renal functions, electrolyte and full blood count. OGTT was performed unless the patient had obvious diabetes. Serum specimens collected at $0,30,60,120$ and $180 \mathrm{~min}$ were used to test GH, glucose, C-peptide and insulin. Urine routine tests were performed and 24-h urinary free cortisol was measured (Details of each assay are provided in Supplementary materials, see section on supplementary data given at the end of this article).

All patients underwent pituitary magnetic resonance imaging examinations. 24-h ambulatory blood pressure monitoring (ABPM), Holter electrocardiogram and Doppler echocardiography were performed to evaluate cardiovascular system. Spirometry and peak flow measurements were used to assess pulmonary functions while polysomnography (PSG) was performed to evaluate sleep apnea. Bone mineral density (BMD) was measured by dual-energy X-ray absorptiometry. Organ volumes were assessed with a 3-dimensional model derived from CT scanning (Details are provided in Supplementary materials). 


\section{Statistical analysis}

Data management and statistical analyses were performed using SPSS Statistics, version 20.0 (IBM). Normally distributed data were displayed as mean \pm S.D., while variables with a non-normally distribution were expressed as median with interquartile range. Variables were compared using repeated-measures ANOVA and LSD post hoc analysis for the differences among each two visits, with $P<0.05$ indicating a statistically significant difference.

\section{Results}

At one-year visit, twenty-four patients were considered to be cured and were included in the final analysis. The baseline characteristics of the 24 patients are presented in Supplementary Table 1 . As the obtained data were too abundant, only those novel data were presented here (All other data were demonstrated in Supplementary materials).

\section{Endocrine system}

Random GH levels dropped immediately from 16.31 $(7.65-48.17) \mu \mathrm{g} / \mathrm{L}$ to $0.50(0.35-0.68) \mu \mathrm{g} / \mathrm{L}$ on the first day postoperatively. Random $\mathrm{GH}, \mathrm{GH}_{\mathrm{n}}$ and IGF-I index dropped dramatically at one week postoperatively (all $P<0.0001$, Fig. 1A, B and C). Normal GH, defined as either a $\mathrm{GH}_{\mathrm{n}}<0.4 \mu \mathrm{g} / \mathrm{L}$ or random $\mathrm{GH}<1.0 \mu \mathrm{g} / \mathrm{L}$, was achieved in $18(75 \%)$ patients at one week, $22(91.67 \%)$ at one month and all by three months. The IGF-I index dropped from $2.59 \pm 0.82$ to $1.28 \pm 0.41$ at one week $(P<0.0001)$. Interestingly, 6 (25\%) patients quickly achieved normal IGF-I at one week while 17 (70.83\%) patients fall within normal range at one month.

\section{Glucose metabolism}

At study entry, 20 (83.33\%) patients exhibited abnormal glucose metabolism. As early as one week after surgery, normal glucose tolerance was restored in 8/20 (40\%) patients. At one month after surgery, only 4 (16.67\%) patients had persistent diabetes (Fig. 1D).

Compared to baseline, fasting glucose at one week and one month after surgery significantly declined $(5.91 \pm 1.21$, $5.15 \pm 1.33$ and $4.72 \pm 0.72 \mathrm{mmol} / \mathrm{L}$, respectively, $P=0.004$ and $P<0.001$ ) (Fig. 1E). Two-hour glucose of OGTT at one month was significantly lower than baseline $(10.33 \pm 4.34$ vs $6.48 \pm 3.49 \mathrm{mmol} / \mathrm{L}, P<0.001$ ) (Fig. $1, \mathrm{E}$ and $\mathrm{F}$ ) and stayed stable onwards. Fasting insulin and insulin levels during OGTT also decreased dramatically at one week and further declined at one month (Fig. 1, G and H). Fasting c-peptide and c-peptide during OGTT decreased at one month (Supplementary Fig. 1F and G). Surprisingly, $\mathrm{HbA}_{1 \mathrm{C}}$ declined as early as one month after the surgery $(6.92 \pm 2.92$ vs $6.02 \pm 1.42 \%, P=0.022)$ and persistently fell until three months $(P=0.025)$ (Fig. 1I).

At one week, HOMA-IR quickly fell from 3.83 $(1.72-6.36)$ at baseline to $1.59(1.08-2.58)(P=0.01)$ at one week (Fig. 1J). With insulin resistance defined as HOMA-IR greater than three, 16 (66.67\%) patients were insulin resistant at baseline while only one (4.17\%) remained resistant at one month. ISOGTT, the index of whole body insulin sensitivity, started increasing from one week (Fig. 1K). Beta cell function was evaluated by insulinogenic index (IGI), INSO/BG0 (Supplementary Fig. 1H and I) and HOMA- $\beta$ (Fig. 1L). Compared with baseline, the three indexes significantly decreased at one week $(P=0.024)$, one month $(P=0.004)$ and 3 months $(P=0.004)$ respectively.

\section{Cardiovascular system}

Compared to baseline, mean heart rate (mHR) in daytime significantly declined at one week, but returned to preoperative levels at three months, while progressively dropped again since then. Nocturnal mHR started decreasing at 6 months (Fig. 2A). 11 patients had hypertension at baseline and 7 were on therapy at baseline. After surgery, only 4 patients showed persistent hypertension and 3 patients received hypertensive medication. Daytime mean systolic blood pressure (mSBP), daytime mean diastolic blood pressure (mDBP) and nocturnal mSBP started to drop at one week (Fig. 2B and C). All patients achieved normal diurnal $\mathrm{mSBP}$, diurnal mDBP as well as nocturnal mSBP at 12 months. Although we observed a decreasing tendency in nocturnal mDBP at the last visit $(72.74 \pm 16.13$ vs $68.33 \pm 7.70 \mathrm{mmHg}$, $P=0.085)$, half of the patients still had high nocturnal $\mathrm{mDBP}(>70 \mathrm{mmHg}$ ) at one-year follow-up (Fig. 2C).

Echocardiography revealed that left ventricular mass (LVM) index and LVM dropped at one year (Fig. 2D and Supplementary Fig. 3E). Compared with baseline, total ejection isovolume (Tei) index, reflecting overall cardiac function, significantly decreased at 12 months $(P=0.043$, Supplementary Fig. 3J). Changes of left ventricular dimensions, systolic function and diastolic function displayed in Supplementary Fig. 3. 
A

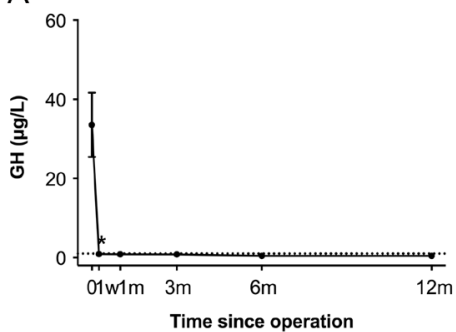

D

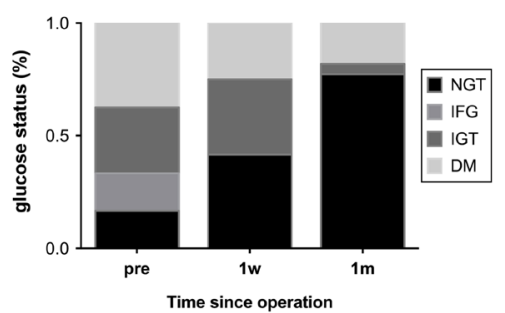

G

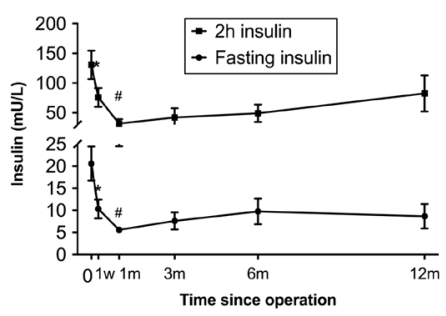

J

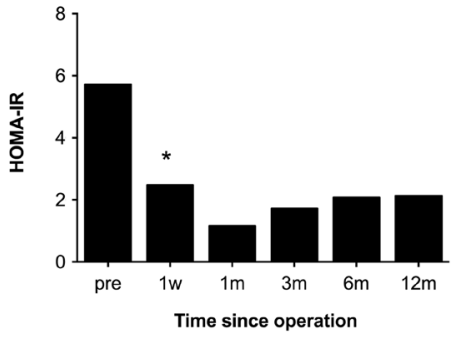

B

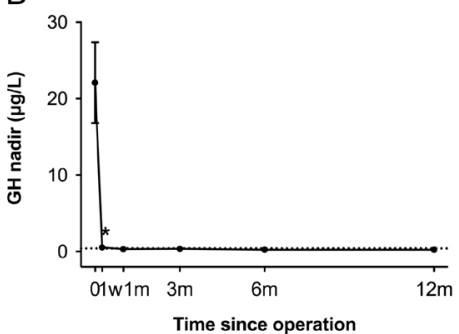

E

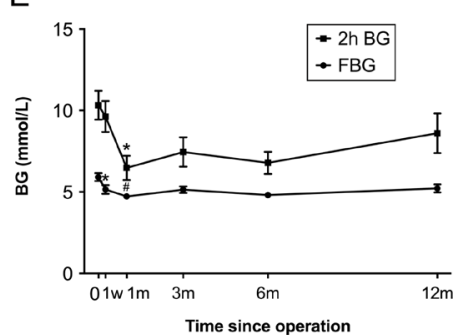

$\mathrm{H}$

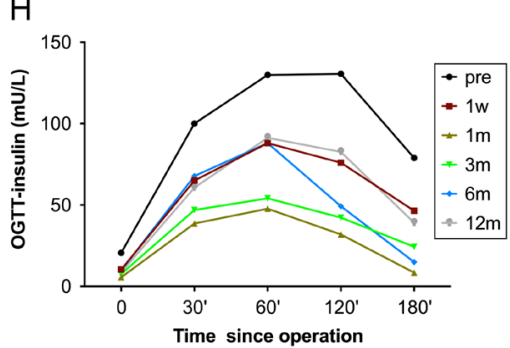

K

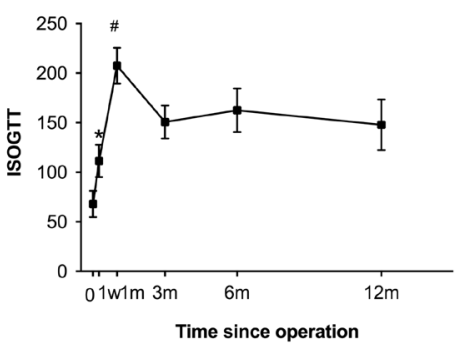

C

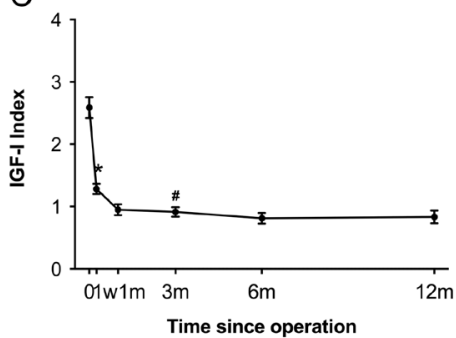

F

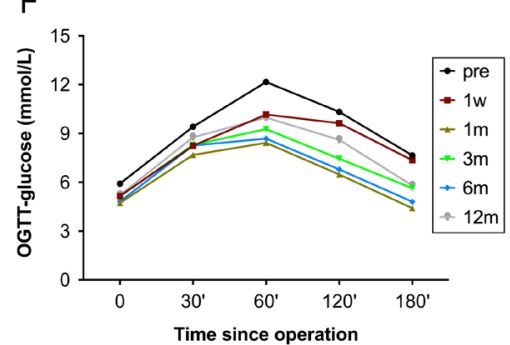

।

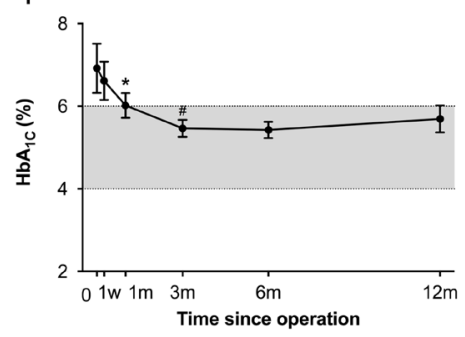

$\mathrm{L}$

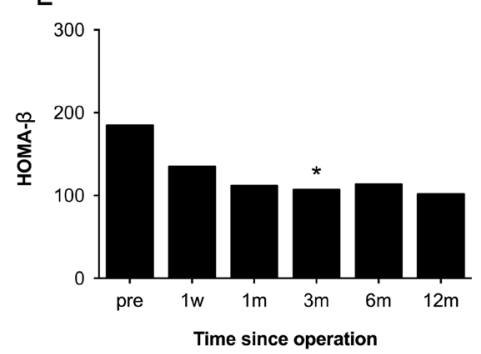

Figure 1

Changes of endocrine system and glucose metabolism. Effects of surgical remission on endocrine system and glucose metabolism in patients with acromegaly. Panel A, B and C: GH, GH nadir and IGF-I index dropped dramatically at one week after surgery. Panel D: Glucose homeostasis improved as early as one week and remained stable since one month. Panel E: Fasting glucose significantly declined at one week and one month after surgery; 2-h glucose of OGTT was significantly lower than baseline at one month and stayed stable onwards. Panel F: AUC for BG dropped since one week and further declined at one month, while there was no difference among months one, three, six and 12. Panel G: Fasting insulin and 2-h insulin decreased at one month after surgery. Panel H: Insulin of OGTT dropped since one week and further declined at one month while there was no difference among later visits. Panel I: HbA1C significantly decreased at one month after the surgery and persistently declined at three months. Panel J: HOMA-IR fell at one week, but there were no differences among later follow-ups. Panel K: ISOGTT increased from one week. Panel L shows HOMA- $\beta$ decreased at three months and remained stable onwards (Data are expressed as mean \pm s.E. ${ }^{*} P<0.05$ compared with preoperative level; ${ }^{*} P<0.05$ compared with one week level; ${ }^{* *} P<0.05$ compared with one month level. The gray shaded area marked the normal range. AUC, areas under curve; BG, blood glucose; FBG, fasting blood glucose; GH, growth hormone; IGF-1, insulin-like growth factor-1; ISOGTT, the index of whole body insulin sensitivity; OGTT, oral glucose tolerance test; $2 \mathrm{~h} \mathrm{BG}, 2$-h blood glucose of OGTT; $2 \mathrm{~h}$ insulin, 2-h serum insulin of OGTT). 
A
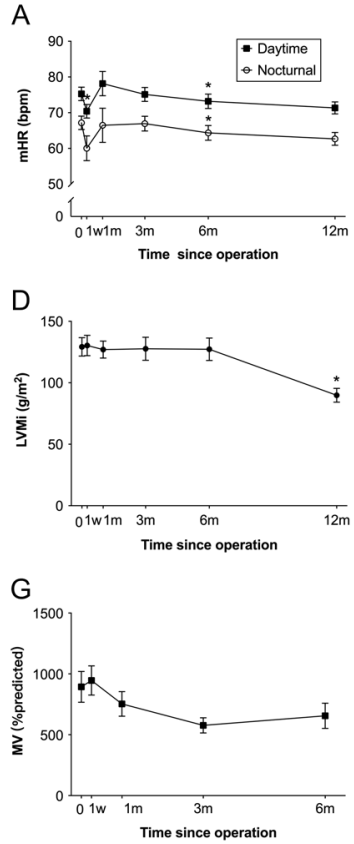

$J$

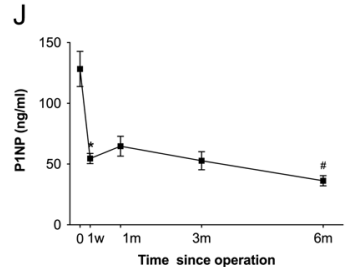

B

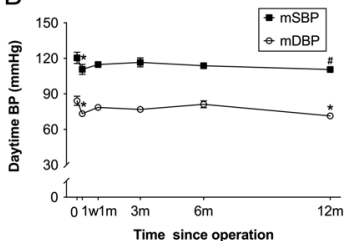

E

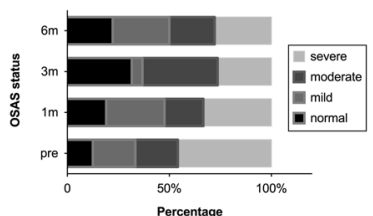

$\mathrm{H}$

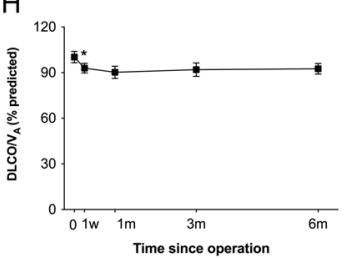

K

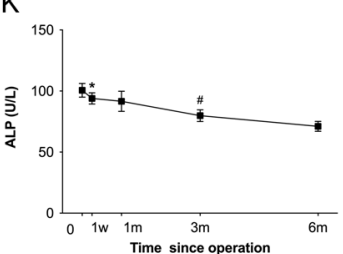

C

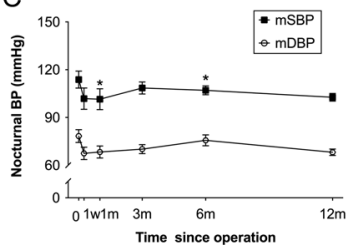

F
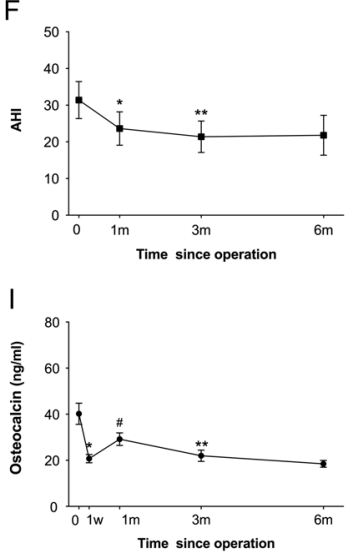

L

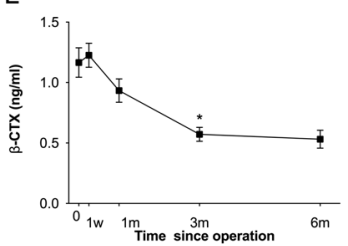

Figure 2

Changes of cardiac function, pulmonary function and bone metabolism markers. Panel A: Daytime mean heart rate (mHR) significantly decreased at one week, but restored to preoperative levels at one month while progressively dropped at later visits. Nocturnal mHR started decreasing at 6 months. Panel B: Daytime mean systolic blood pressure (mSBP) and daytime mean diastolic blood pressure (mDBP) dropped quickly at one week and remained at low levels at the following visits. Panel C: Nocturnal mSBP started to drop at one week and got significantly lower since six months. Nocturnal mDBP displayed a slightly decreasing tendency at 12 months. Panel D: LVMi decreased significantly at 12 months postoperatively. Panel E: OSAS status got improved from one month postoperatively. Panel F: AHI dropped dramatically at one month, further declined at three months and got stable then. Panel G \& H: MV was remarkably high at baseline and was unchanged during the whole follow-up. DLCO/VA significantly decreased from one week postoperatively. Panel I, J and K shows bone formation markers including N-Mid osteocalcin, P1NP and ALP decreased rapidly at one week after surgery and progressively declined till six months. Panel $L$ shows bone resorption marker $\beta$-CTX significantly decreased at three months postoperatively. (Data are expressed as mean \pm S.E. ${ }^{*} P<0.05$ compared with preoperative level; ${ }^{\#} P<0.05$ compared with one week level; ${ }^{*} P<0.05$ compared with one month level. AHI, apnea hypopnea index; ALP, serum alkaline phosphatase; DLCO/VA, diffusion capacity for carbon monoxide/ventilation; LVMi, left ventricular mass index; MV, minute ventilation; OSAS, obstructive sleep apnea syndrome; P1NP, procollagentype $1 \mathrm{~N}$-terminal propeptide; $\beta$-CTX, $\beta$-isomerof C-terminal telopeptide of type I collagen).

PSG

Sleep apnea syndrome (SAS) was seen in 21 patients $(87.5 \%)$ at baseline, with 20 patients having obstructive sleep apnea syndrome (OSAS) and 1 patient with central sleep apnea. At six months, 11 patients (55\%) with OSAS showed improvement. The percentage of severe OSAS decreased from $45.8 \%$ at baseline to $28 \%$ at six months (Fig. 2E). Mean apnea-hypopnea index (AHI) dropped dramatically at one month $(P=0.019)$, and further declined at three months $(P=0.006)$ and got stable since then (Fig. 2F), which paralleled with 
the changes of apnea index (AI) (Supplementary Fig. 4A). By six months, other indexes for sleep quality including total awake time, awakening numbers and sleep efficacy were improved $(P=0.06,0.08$, and 0.055 respectively), despite no statistical significance reached (Supplementary Fig. 4B, C and D). Hypopnea index and total sleep time showed no change within 6 months (Supplementary Fig. 4E and F).

\section{Pulmonary function}

The mean total lung capacity (TLC) \% predicted was $116 \pm 28 \%$ at baseline and 10 patients had large lungs. Other parameters of lung volumes and ventilation function were also elevated in acromegaly than in general population. By six months, all these indexes showed no significant change (Fig. 2G and Supplementary Fig. 5A, B, C, D, E, F, G and H).

DLCO $\%$ and DLCO $/ \mathrm{V}_{\mathrm{A}} \%$, reflecting diffusion capacity, significantly decreased at three months (Supplementary Fig. 5I) and one week, respectively (Fig. 2H). Respiratory muscle hypofunction was detected at baseline, reflected by MIP\%, MEP\% and P0.1/MIP\% (35 $\pm 18 \%, 51 \pm 22 \%$, $609 \pm 529 \%)$, and this status was not improved (Supplementary Fig. 5J, K and L).

\section{Bone metabolism}

Bone formation markers, including N-mid osteocalcin, procollagen type $1 \mathrm{~N}$-terminal propeptide (P1NP) and serum alkaline phosphatase (ALP), dropped rapidly at one week after surgery $(P<0.001, P<0.001$, and $P=0.008)$ and declined until six months (Fig. 2I, J and K). However, $\beta$-isomer of C-terminal telopeptide of type I collagen ( $\beta$ CTX), the bone resorption marker, significantly dropped at three months (Fig. 2L). BMD of the lumbar and hip showed no change during the follow-up (Supplementary Fig. 6E, F, G and H).

\section{Morphological changes}

Compared to baseline, finger joint circumference, hand volume, the height of the lower lip and nasal ala fell at one week and persistently declined during the entire follow-up, while foot size became smaller starting at three months (Table 1).

The cross-sectional area of tongue persistently decreased starting at one month ( $P=0.001$, Fig. $3 \mathrm{~A}$, B and $C)$, accompanied with the airway volume getting larger $(P=0.049$, Table 1$)$. Liver, spleen and kidney volumes

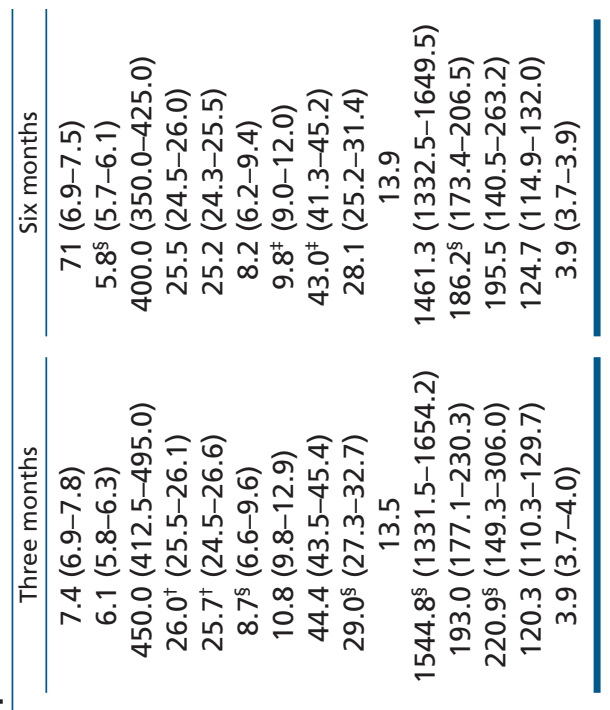


declined by $6.4,9.2,15.9 \%$, respectively at one month and continuously went down at six months $(P=0.009$, $P<0.001$, and $P<0.001$ ) (Fig. $3 \mathrm{D}, \mathrm{E}, \mathrm{F}, \mathrm{G}, \mathrm{H}$ and $\mathrm{I}$ and Table 1). The pancreas volume at baseline was larger than that in general population and remained unchanged during the entire follow-up ( $P=0.818$, Table 1$)$, which has not been reported previously (9). Interestingly, the adrenal volume was similar with that of the general population in China (10). However, adrenal lesions including nodules and hyperplasia were identified in 10 patients (41.67\%). The lesions showed no change during the follow-up.

In the current study, we observed that some parameters changed rapidly and dramatically after surgery (Fig. 4A) while some variables slowly and mildly fell throughout the entire follow-up (Fig. 4B). However, other parameters presented no change at all (Fig. 4C).

\section{Discussion}

To our knowledge, this is the first study which prospectively investigated the dynamic and systemic changes in anatomy and function induced by the sudden resolution of GH excess and characterized organ- and time-dependent amelioration of acromegaly-related comorbidities. The various changes observed emphasize that excessive GH and IGF-I have complex effects on the structure and function of organs, while the reversibility of excessive GH-induced comorbidities is time dependent and varies among organs.

We found that in patients with acromegaly who achieved remission after surgery, the random GH levels dropped rapidly on the first day and became stable at one week postoperatively. After the quick GH resolution, the synthesis and secretion of IGF-I decreased, resulting in the rapid fall of serum IGFI. Thus, the postoperative changing pattern of IGF-I levels was characterized by a marked decrease in the first week after adenomectomy, followed by a gradual stabilization to the defined values of remission. It is generally accepted that the optimal postoperative timing for assessing IGF-I is eight to 12 weeks (8). However, in our cohort, 25\% patients and 70.83\% patients achieved normal IGF-I at one week and one month, respectively. Rapid decline of serum IGF-I during the immediate postoperative period warrants further study, which examines the value of IGF-I as an early predictor for biochemical remission (11).

The rapid decline of $\mathrm{GH}$ led to immediate improvement of glucose metabolism one week after surgery and complete remission occurred at one month, which has not been reported. This quick restoration is attributed to the improved insulin resistance. Therefore, we suggest that blood glucose should be closely monitored within a few weeks after surgery, especially for those treated with anti-diabetic medications. Due to the quick restoration, patients may need dose adjustment in order to avoid hypoglycemia. However, if diabetes persists after one month of surgical remission, it may be caused by other pathological reasons or irreversible due to long-term exposure to excessive $\mathrm{GH}$.

Cardiovascular comorbidities contribute significantly to mortality in acromegaly (1). Here, we provided detailed evaluations showing that heart rate significantly decreased only at six months, which was far behind the decline of GH and IGF-I. The delayed improvement in HR may be due to the persistent hyperkinetic syndrome and slow recovery from the $\mathrm{GH}$ action on cardiac conduction system and sympathovagal balance (12). Evaluated by ABPM, hypertension was present in $53.85 \%$ patients at baseline, which was much higher than that in general population in China (13). Further, we revealed that it predominantly involved high diastolic BP, which is more difficult to manage. Surprisingly, nocturnal mDBP was unaltered during the entire follow-up and half of the patients still presented high nocturnal DBP at the one-year visit. These novel findings suggest that excessive GH exposure may lead to irreversible damage to arterial walls, and more research is needed to explore the potential mechanisms. A sharp decrease of daytime BP combined with a blunted nocturnal BP decline resulted in the disturbed circadian $\mathrm{BP}$, as shown by highly prevalent non-dipping profile in our cohort. The high nocturnal DBP and a disturbed BP circadian rhythm represent cardiovascular risks (14). Therefore, we strongly recommend that 24-h ABPM, which helps to detect high nocturnal DBP, should be routinely undertaken in patients with acromegaly. Changes of left ventricular dimensions, systolic function and diastolic function were consistent with previous studies. The indices for cardiac hypertrophy, systolic function and diastolic function only changed at 12 months after surgical remission, suggesting the slow recovery of cardiac structures and function. Therefore, long-term evaluation of cardiac structure and function is needed, even after GH normalization.

Respiratory disorders contribute to $25 \%$ of all deaths recorded in acromegaly (1). However, data about the effects of surgical remission on respiratory function are scarce. For the first time, we report that despite of GH normalization, pulmonary function showed no 
A

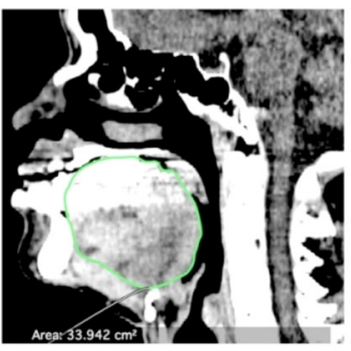

B

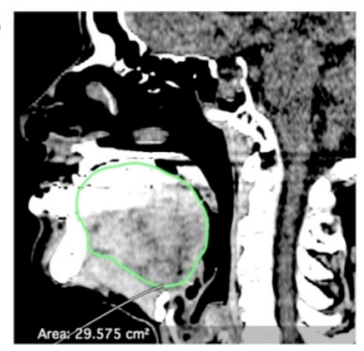

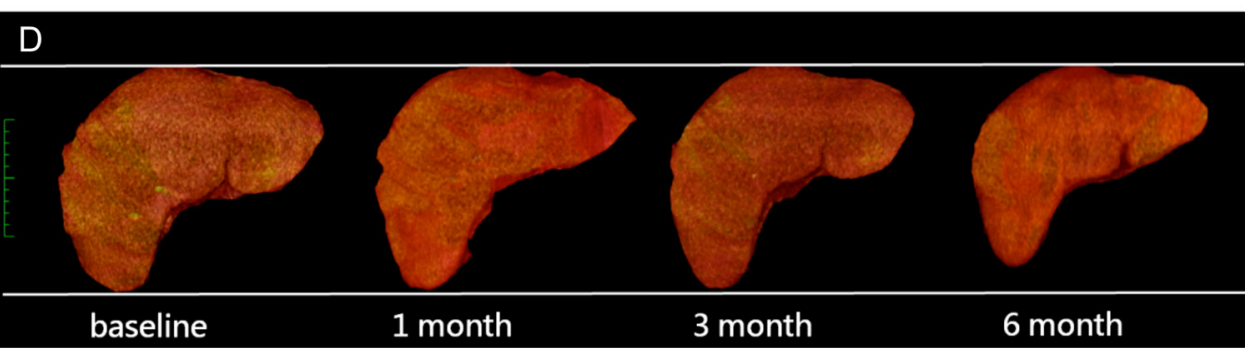
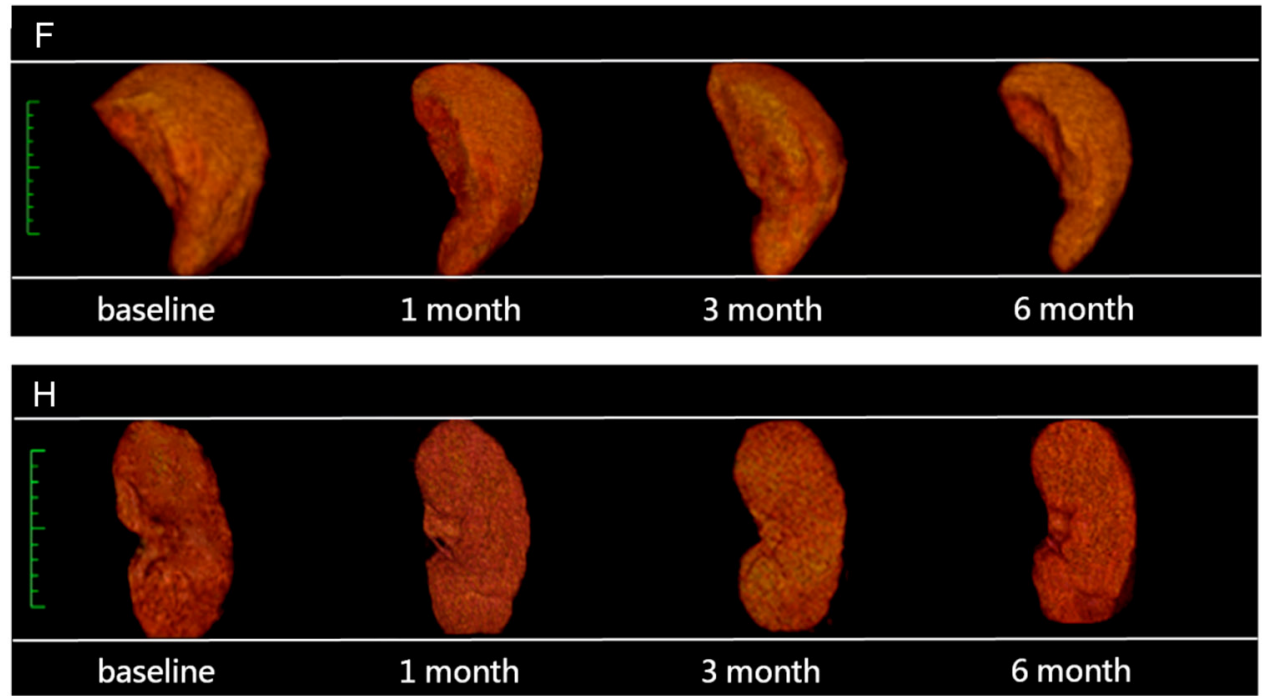

C

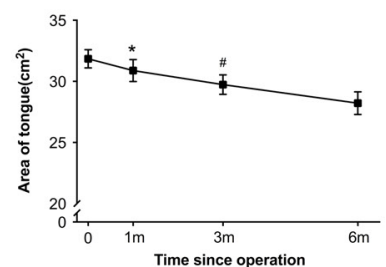

E

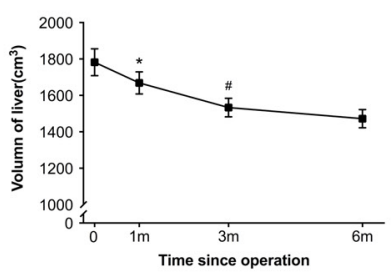

G
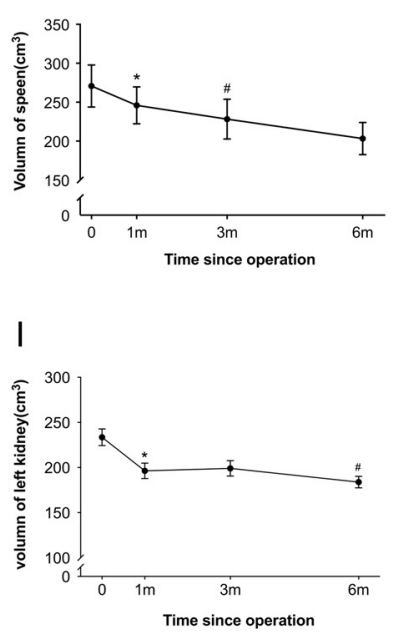

\section{Figure 3}

Changes of the tongue area and organ volumes. Panel A, B and C show the changes of tongue area. Compared with the baseline, tongue area decreased since one month postoperatively. Panel D and E show the reconstructed liver size immediately got smaller at one month, and persistently went down at 6 months. Panel F and G show the volume of spleen progressively declined during the follow-up. Panel $\mathrm{H}$ and I show the reconstructed kidney size persistently declined. (Data are expressed as mean \pm S.E. ${ }^{*} P<0.05$ compared with the preoperative visit; ${ }^{\#} P<0.05$ compared with one month.).

significant improvement within one year. We speculate that in addition to GH and IGF-I, the recovery of pulmonary function is determined by multiple other factors. Therefore, although $\mathrm{GH}$ resolution leads to the alleviation of other comorbidities, impairment in pulmonary function persists and long-term evaluation is required. Long-term follow-up and evaluation are required to observe the changes in lung function. Additionally, we demonstrate that GH resolution leads to improved obstructive sleep apnea syndrome (OSAS) and enhanced sleep architecture. Surprisingly, the benefits occurred as early as one month after surgery, which was not reported previously. This is partially attributed to the smaller tongue area and larger airway volume, as reported in our results. Noticeably, no patients with severe OSAS recovered to normal within six months postoperatively. Thus, these patients still need treatment even after biochemical remission.

GH and IGF-I are important regulators of bone growth modeling and remodeling during life span 

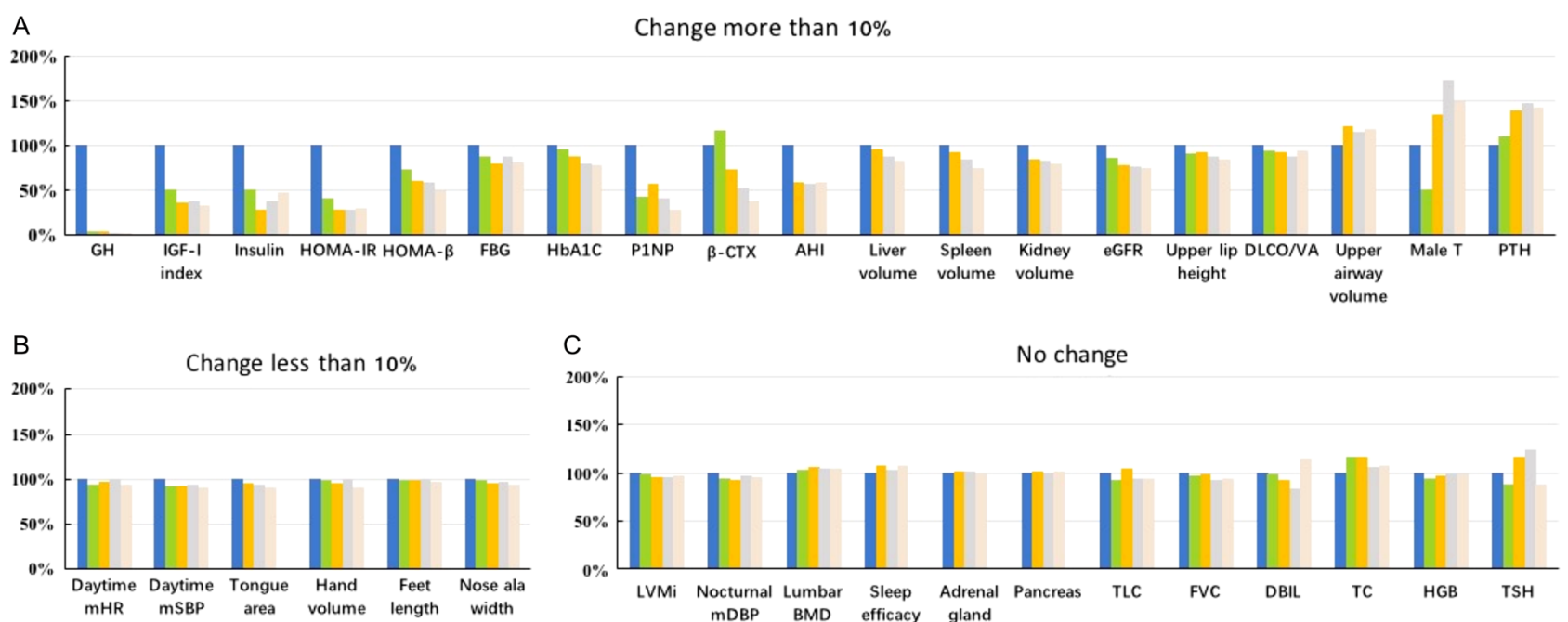

C

No change

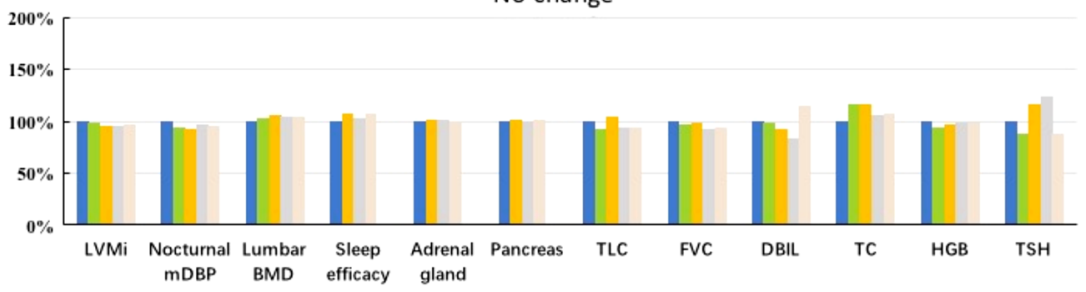

abseline $=1$ week 1 month $=3$ months 6 months

\section{Figure 4}

Dynamic and systemic changes after resolution of $\mathrm{GH}$. This figure highlights the different systemic changes induced by the quick GH resolution. Panel A shows major parameters with more than $10 \%$ changes, including GH, IGF-I index, insulin, HOMA-IR, HOMA- $\beta, F B G, H_{1} A_{1 C}$ P1NP, $\beta-C T X, A H I$, liver volume, spleen volume, kidney volume, eGFR, upper lip height, DLCO/N ${ }_{A}$, upper airway volume, male testosterone (T) and PTH. Panel B shows parameters with significant but less than $10 \%$ changes, including daytime $\mathrm{mHR}$, daytime $\mathrm{mSBP}$, tongue area, hand volume, feet length, and nose ala width. Panel $\mathrm{C}$ shows parameters without significant changes, including LVMi, nocturnal mDBP, lumbar BMD, sleep efficacy, adrenal gland volume, pancreas volume, TLC, FVC, DBIL, TC, HGB, and TSH. (Data are expressed as mean. AHI, apnea hypopnea index; BMD, bone mineral density; DBIL, direct bilirubin; $\mathrm{DLCO} / \mathrm{N}_{\mathrm{A}}$, diffusion capacity for carbon monoxide/ventilation; eGFR, estimated glomerular filtration rate; FBG, fasting blood glucose; FVC, forced vital capacity; GH, growth hormone; HGB, hemoglobin; IGF-I, insulin-like growth factors-l; LVMi, left ventricular mass index; $\mathrm{mDBP}$, mean diastolic blood pressure; $\mathrm{mHR}$, mean heart rate; $\mathrm{mSBP}$, mean systolic blood pressure; $\mathrm{P} 1 \mathrm{NP}$, procollagentype I N-terminal propeptide; PTH, parathyroid hormone; TC, total cholesterol; TLC, total lung capacity; TSH, thyroidstimulating hormone; $\beta$-CTX, $\beta$-isomerof C-terminal telopeptide of type I collagen).

(15). Bone turnover is increased in active acromegaly, suggesting activation of both osteoblasts and osteoclasts (16). We demonstrated that the decline of bone formation markers occurred as early as one week after remission while the bone resorption parameters dropped significantly at three months. The changes of bone formation occurred earlier than bone resorption, which was opposite to a previous report (17). Tamada and coworkers reported that bone resorption markers decreased at three months while bone formation markers decreased at 12 months after surgery (18). The disparity may result from the different markers used and the shorter follow-up interval in our study helped to detect earlier changes. Our data support previous hypotheses that GH and IGF-I acted as anabolic hormones by stimulating bone turnover, especially bone formation, and thus, the bone formation changed earlier (19). Claessen and coworkers indicated a high prevalence of vertebral fractures in patients with acromegaly despite long-term remission (20). Thus, better approaches to detect bone metabolism is necessary, since the current dual-energy X-ray absorptiometry does not reflect all the bone metabolism parameters and bone health.

Patients with acromegaly generally present with typical appearances including coarsened facial features, enlarged hands and feet, as well as soft tissue hypertrophy. Few studies had comprehensively described the dynamic changes after remission. Here, we demonstrated that nose, lips and hands became smaller as soon as one week postoperatively, while the change in feet occurred at three months. Besides these readily visible changes, what is more remarkable is the time course of reversible changes of enlarged internal organs. The tongue and upper airway showed significant change at one month, which may result in the improvement of sleep status. Liver, kidney and spleen volumes decreased markedly at one month. 
Interestingly, the enlarged pancreas showed no change at all, which suggested that either it takes longer time for the pancreas to recover or it is irreversible. Additionally, we here reported patients with acromegaly had higher prevalence of adrenal nodules/hyperplasia (41.67\%) than in the general population (0.5-5\%) (21), which may be attributed to the potent mitogenic and proliferative effect of excessive GH and IGF-I.

The distinct responses of different systems to quick GH resolution are of interests but the mechanisms are unknown, probably the time of GH excess in different organs plays an important role. Finally, we observed inexplicable fluctuation in some parameters during the follow-up, which may be affected by multiple factors, and further investigations are needed to reveal the mechanisms underlying those interesting phenomenon. Such as the fluctuation of the daytime mHR and blood pressures in the first month after the surgery may due to the changes in the volumes of the blood and the medicine used in the perioperative period.

For the first time, we described the comprehensive and detailed changes of the whole body after GH resolution, while lacking mechanistic investigation. Our study demonstrated the distinct effects of lowering GH and IGF-I on various systems and showed the time course for those reversible changes. The quick restoration of excessive GH- and IGF-I-induced systemic changes in a time- and organ-dependent manner, which not only helps to understand the pathophysiological effects of excessive GH on human body, but also make plans for follow-up management. Our novel findings can inform future research and patient care in multiple relevant areas. In addition, we found that some impaired function and structures are potentially irreversible (at least within the time frame of our study), cautioning that active acromegaly may cause permanent damage. Patients with acromegaly even after surgically cured should be carefully and closely followed up.

There are some limitations of our study. First, as acromegaly is a rare disease, the patient number was small in our study. Second, healthy controls were lacking and further studies with the matched healthy subjects are warranted to support our conclusions. Additional longterm prospective investigations in other population are also needed to confirm our results.

\section{Supplementary data}

This is linked to the online version of the paper at https://doi.org/10.1530/ EJE-17-0872.
Declaration of interest

The authors declare that there is no conflict of interest that could be perceived as prejudicing the impartiality of this study.

\section{Funding}

This work was supported by the China Pituitary Adenoma Specialist Council (CPASC) and has received funding from the Chang Jiang Scholars Program, the National Program for Support of Top-Notch Young Professionals, the Program for New-Century Excellent Talents in University (NCET-10-0356), the Shanghai Rising-Star Tracking Program (12QH1400400), the National High Technology Research and Development Program of China (2014AA020611), the National Natural Science Foundation of China (81172391), Shanghai Municipal Commission of Health and Family Planning (20134280) to Yao Zhao; National Foundation and Major Basic Research Program of Shanghai (16JC1420100) to Ying Mao. The funders had no role in study design, data collection and analysis, decision to publish, or preparation of the manuscript.

\section{Author contribution statement}

Z Y Z, Q L, W Q H, Q H J and Y Z contributed to data collection, data analysis, data interpretation and writing of the report. $Z Y Z, Q L, Q H J$ and $Y Z$ contributed to study design. $H Y Y, Y F W, M S, M H, Y F Y, S X F$ and $\mathrm{HCX}$ contributed to data analysis and data interpretation. $\mathrm{HY}, \mathrm{GQH}$, W J T, D Y G, C J L, Z Y M, Z Y, Q L Z, Y C Z, Y S, Y P Y, M W, X D L and Y L contributed to data collection. CW F designed the statistical method. S Q L contributed to study design and reviewed the report. N A T contributed to data interpretation and writing of the report. $\mathrm{R} \mathrm{M} \mathrm{H,} \mathrm{Y} \mathrm{M,} \mathrm{L} \mathrm{F} \mathrm{Z} \mathrm{and} \mathrm{Y} \mathrm{M} \mathrm{L}$ contributed to study design and coordination as center leader.

\section{Acknowledgments}

The authors thank and acknowledge all of the participants in the study. They gratefully thank Min Zhu for statistical analysis, Zhihua Zhang, Linlin Yan, Xiaolan Lu and Xiaotian Sun for data collection and analysis. They thank Dr Robert Stanton for helpful comments and critical reading of the manuscript.

\section{References}

1 Colao A, Ferone D, Marzullo P \& Lombardi G. Systemic complications of acromegaly: epidemiology, pathogenesis, and management. Endocrine Reviews 200425 102-152. (https://doi. org/10.1210/er.2002-0022)

2 Melmed S. Medical progress: acromegaly. New England Journal of Medicine 2006355 2558-2573. (https://doi.org/10.1056/ NEJMra062453)

3 Webb SM, Casanueva F \& Wass JA. Oncological complications of excess GH in acromegaly. Pituitary 20025 21-25. (https://doi. org/10.1023/A:1022149300972)

4 Dutta P, Bhansali A, Vaiphei K, Dutta U, Ravi Kumar P, Masoodi S, Mukherjee KK, Varma A \& Kochhar R. Colonic neoplasia in acromegaly: increased proliferation or deceased apoptosis? Pituitary 201215 166-73. (https://doi.org/10.1007/s11102-011-0300-9)

5 Dworakowska D, Gueorguiev M \& Kelly P. Repeated colonoscopic screening of patients with acromegaly: 15-year experience identifies those at risk of new colonic neoplasia and allows for effective screening guidelines. European Journal of Endocrinology 2010163 21-28 (https://doi.org/10.1530/EJE-09-1080) 
6 Minniti G, Moroni C, Jaffrain-Rea ML, Esposito V, Santoro A, Affricano C, Cantore G, Tamburrano G \& Cassone R. Marked improvement in cardiovascular function after successful transsphenoidal surgery in acromegalic patients. Clinical Endocrinology 200155 307-313. (https://doi.org/10.1046/j.13652265.2001.01343.x)

7 Colao A, Cuocolo A, Marzullo P, Nicolai E, Ferone D, Della Morte AM, Pivonello R, Salvatore M \& Lombardi G. Is the acromegalic cardiomyopathy reversible? Effect of 5-year normalization of growth hormone and insulin-like growth factor I levels on cardiac performance. Journal of Clinical Endocrinology and Metabolism 200186 1551-1557. (https://doi.org/10.1210/jcem.86.4.7376)

8 Giustina A, Chanson P, Bronstein MD, Klibanski A, Lamberts S, Casanueva FF, Trainer P, Ghigo E, Ho K \& Melmed S. A consensus on criteria for cure of acromegaly. Journal of Clinical Endocrinology and Metabolism 201095 3141-3148. (https://doi.org/10.1210/jc.2009-2670)

9 Saisho Y, Butler AE, Meier JJ, Monchamp T, Allen-Auerbach M, Rizza RA \& Butler. PC. Pancreas volumes in humans from birth to age one hundred taking into account sex, obesity, and presence of type 2 diabetes. Clinical Anatomy 200720 933-942. (https://doi. org/10.1002/ca.20543)

10 Zou H, Chen Y \& Yang G. Image anatomy of adult adrenal gland. Chinese Journal of Anatomy 200831 96-98. (https://doi,org/10.3969/j. issn.1001-1633.2008.01.028)

11 Yu M, Bruns DE, Jane JA Jr, Nass RM, Oldfield EH, Vance ML \& Thorner MO. Decrease of serum IGF-I following transsphenoidal pituitary surgery for acromegaly. Clinical Chemistry 201763 486-494. (https://doi.org/10.1373/clinchem.2016.262592)

12 Comunello A, Dassie F, Martini C, De Carlo E, Mioni R, Battocchio M, Paoletta A, Fallo F, Vettor R \& Maffei P. Heart rate variability is reduced in acromegaly patients and improved by treatment with somatostatin analogues. Pituitary 201518 525-534. (https://doi.org/10.1007/s11102-014-0605-6)

13 Wu Y, Huxley R, Li L, Anna V, Xie G, Yao C, Woodward M, Li X, Chalmers J, Gao R et al. Prevalence, awareness, treatment, and control of hypertension in China: data from the China National Nutrition and Health Survey 2002. Circulation 2008118 2679-2686. (https://doi.org/10.1161/CIRCULATIONAHA.108.788166)
14 Hansen T, Li Y, Boggia J, Thijs L, Richart T \& Staessen J. Predictive role of the nighttime blood pressure. Hypertension 2011 57 3-10. (https://doi.org/10.1161/HYPERTENSIONAHA.109.133900)

15 Claessen KM, Kroon HM, Pereira AM, Appelman-Dijkstra NM, Verstegen MJ, Kloppenburg M, Hamdy NA \& Biermasz NR. Progression of vertebral fractures despite long-term biochemical control of acromegaly: a prospective follow-up study. Journal of Clinical Endocrinology and Metabolism 201398 4808-4815. (https:// doi.org/10.1210/jc.2013-2695)

16 Ezzat S, Melmed S, Endres D, Eyre DR \& Singer FR. Biochemical assessment of bone formation and resorption in acromegaly. Journal of Clinical Endocrinology and Metabolism 199376 1452-1457. (https:// doi.org/10.1210/jcem.76.6.8501150)

17 Tamada D, Kitamura T, Takahara M, Oshino S, Saitoh Y, Otsuki M \& Shimomura I. Postoperative changes in bone metabolism and bone mineral density in Japanese patients with acromegaly: a 3-year prospective study. Endocrine Journal 201562 1031-1036. (https://doi. org/10.1507/endocrj.EJ15-0174)

18 Tamada D, Kitamura T, Onodera T, Tabuchi Y, Fukuhara A, Oshino S, Saitoh Y, Hamasaki T, Otsuki M \& Shimomura I. Rapid decline in bone turnover markers but not bone mineral density in acromegalic patients after transsphenoidal surgery. Endocrine Journal 201461 231-237. (https://doi.org/10.1507/endocrj.EJ130387)

19 Giustina A, Mazziotti G \& Canalis E. Growth hormone, insulin-like growth factors, and the skeleton. Endocrine Reviews 200829 535-559. (https://doi.org/10.1210/er.2007-0036)

20 Wassenaar MJ, Biermasz NR, Hamdy NA, Zillikens MC, van Meurs JB, Rivadeneira F, Hofman A, Uitterlinden AG, Stokkel MP, Roelfsema F et al. High prevalence of vertebral fractures despite normal bone mineral density in patients with long-term controlled acromegaly. European Journal of Endocrinology 2011164 475-483. (https://doi. org/10.1530/EJE-10-1005)

21 Bovio S, Cataldi A, Reimondo G, Sperone P, Novello S, Berruti A, Borasio P, Fava C, Dogliotti L, Scagliotti GV et al. Prevalence of adrenal incidentaloma in a contemporary computerized tomography series. Journal of Endocrinological Investigation 200629 298-302. (https://doi.org/10.1007/BF03344099)

Received 21 October 2017

Revised version received 8 January 2018

Accepted 30 January 2018 